\title{
Optimal finite-difference operators for arbitrarily sampled data
}

\section{Journal Article}

\section{Author(s):}

Koene, Erik F. M.; Robertsson, Johan O.A.

Publication date:

2020-05-01

Permanent link:

https://doi.org/10.3929/ethz-b-000412036

Rights / license:

In Copyright - Non-Commercial Use Permitted

\section{Originally published in:}

GEOPHYSICS 85(3), https://doi.org/10.1190/geo2019-0081.1 


\section{GHzürich}

\section{Research Collection}

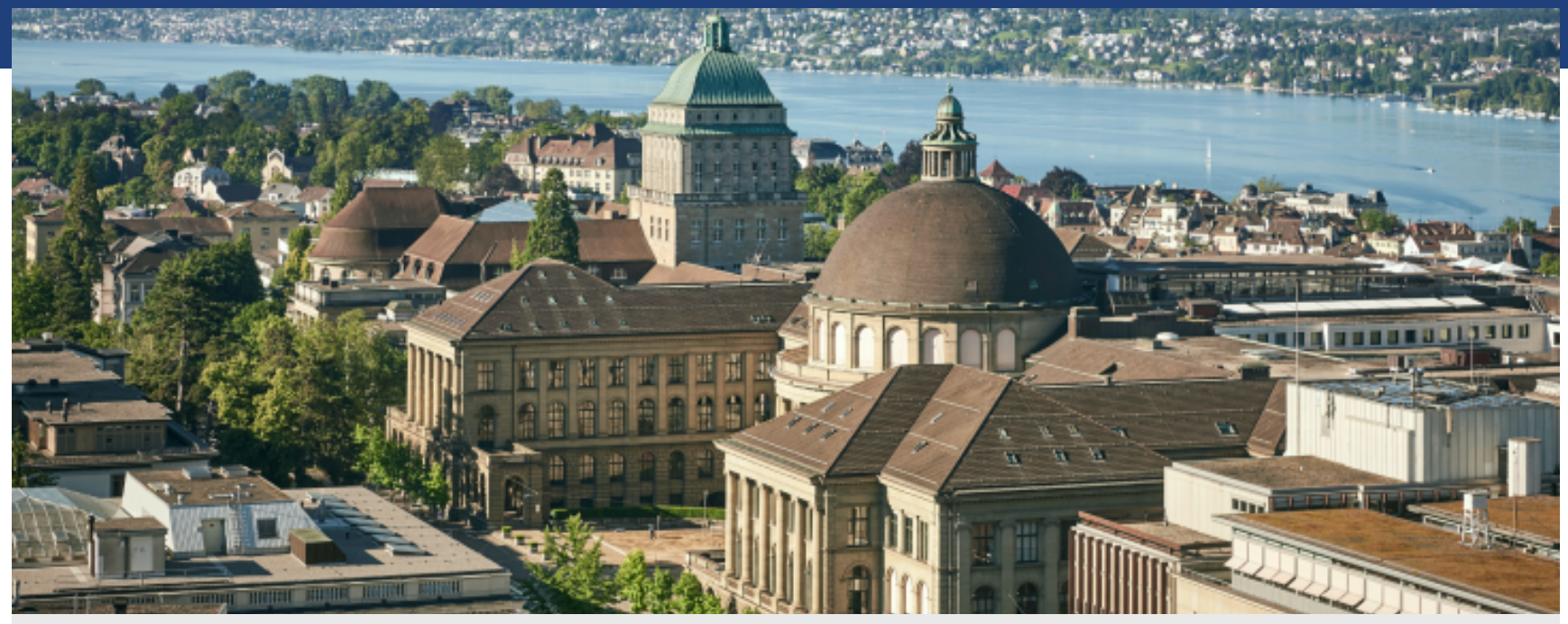

Journal Article

\section{Optimal finite-difference operators for arbitrarily sampled data}

Author(s):

Koene, Erik F. M.; Robertsson, Johan O. A.

Publication Date:

2020-05-01

Originally published in:

GEOPHYSICS 85(3), http://doi.org/10.1190/geo2019-0081.1 $\rightarrow$

Rights / License:

In Copyright - Non-Commercial Use Permitted $\rightarrow$ 


\title{
Optimal finite-difference operators for arbitrarily sampled data
}

\author{
Erik F. M. Koene ${ }^{1}$ and Johan O. A. Robertsson ${ }^{1}$
}

\begin{abstract}
We have developed a general method to obtain the equiripple and the least-squares finite-difference (FD) operator weights to compute arbitrary-order derivatives from arbitrary sample locations. The method is based on the complex-valued Remez exchange algorithm applied to three cost functions: the total error, the relative error, and the group-velocity error. We evaluate the method on three acoustic FD modeling examples. In the first example, we assess the accuracy obtained with the optimal coefficients when propagating acoustic waves through a medium. In the second example, we propagate a wave through an irregular grid. In the final example, we position a source and receiver at arbitrary locations in-between the modeling grid points. In the examples using regular grids, the equiripple solution to the relative cost function performs best. It obtains marginally $(4 \%-10 \%)$ better results compared to the second-best option, the least-squares solution for the relative cost function. The least-squares solution for the relative error produced the only stable and accurate results also in the example of modeling on an irregular grid.
\end{abstract}

\section{INTRODUCTION}

With proper sampling, any band-limited physical field can be reconstructed exactly at arbitrary locations (Robertsson et al., 2008). But when a field is sampled in a less-than-appropriate manner, it can only be approximated with, e.g., constraints on sparsity $\left(L^{0}\right.$ and $L^{1}$-optimality), error energy ( $L^{2}$-optimality), or maximum error ( $L^{\infty}$-optimality). Various algorithms exist to optimize these criteria with varying associated computational costs (Marvasti, 2001; Ikelle and Amundsen, 2018). The finite-difference (FD) operator is one of the cheapest approximation methods, applicable in particular when computational speed is important, and when only a limited number of samples are available.

An FD operator weighs multiple samples to approximate a function or its derivatives at a specific point. To design the operator, an explicit function must be chosen for which the operator should function optimally. Optimizing the operator for polynomial functions yields the classic Taylor coefficients (Fornberg, 1988, 1998; Khan and Ohba, 2000; Bowen and Smith, 2005), whereas optimizing for rational functions yields the Padé approximants (Lele, 1992; Liu and Sen, 2009; Kosloff et al., 2010). For geophysical problems, however, it is more appropriate to obtain operators optimized for periodic functions because any band-limited field is a sum of periodic functions up to a given frequency. One can then either argue that the optimal FD operator should apply to the largest possible bandwidth with the smallest possible error, or that it should have the smallest possible integrated error over this bandwidth. The first requirement is $L^{\infty}$-optimal and results in equiripple operators with oscillating errors. The second requirement results in $L^{2}$-optimal weights. Which of these two optimality criteria yields the best results is a question that is not settled in the literature and may vary per application. Nonetheless, the determination of FD weights for band-limited functions is then done by windowing of the desired band-limited frequency response (Zhou and Greenhalgh, 1992; Hicks, 2002; Chu and Stoffa, 2012; Sun et al., 2015; Zhi-Yang et al., 2015), by $L^{2}$ optimization (Liu, 2013, 2014; Stork, 2013) or by $L^{\infty}$ optimization (Holberg, 1987; Kindelan et al., 1990, 2016; Mittet and Arntsen, 1999, 2000; Zhang and Yao, 2013a, 2013b; Yang et al., 2017; He et al., 2019).

Except for Mittet and Arntsen (1999, 2000) and Hicks (2002), the band-limited operators referenced above are designed only for symmetric operators that have an equal number of samples on both sides of the evaluation point. In contrast, these authors design asymmetric operators that allow one additional sample on either side of the operator, and the authors attempt to produce $L^{\infty}$-optimal solutions. The method of Mittet and Arntsen $(1999,2000)$ is, however, somewhat unclear to implement because details are missing about how to solve the complex-valued least-squares optimization problem for

Peer-reviewed code related to this article can be found at http://software.seg.org/2020/0004

Manuscript received by the Editor 15 February 2019; revised manuscript received 6 August 2019; published ahead of production 12 February 2020; published online 31 March 2020.

ETH Zürich, Institute of Geophysics, Zürich, Switzerland. E-mail: erik.koene@erdw.ethz.ch (corresponding author); johan.robertsson@erdw.ethz.ch.

(C) 2020 Society of Exploration Geophysicists. All rights reserved. 
real-valued coefficients. Furthermore, the operators are only designed to be $L^{2}$ to $L^{6}$-optimal, rather than $L^{\infty}$-optimal. The method of Hicks (2002) is simple to implement but it only applies to an odd number of equidistantly sampled data because it relies explicitly on a windowed version of the sinc interpolator.

In this paper, we derive equiripple and least-squares FD operators for regularly and irregularly sampled data using the Remez exchange algorithm (Remez, 1934; McClellan et al., 1973) extended to the complex domain (Preuss, 1987; Schulist, 1990; Alkhairy, 1994). The operators derived from this method allow, for example, accurate seismic modeling and arbitrary placement of sources and receivers in an FD simulation. The latter can also be found in Mittet and Arntsen (1999, 2000) and Hicks (2002), but, here, we solve the exact optimization problem rather than an approximation of it. Other potential applications are, for example, high-order accurate modeling of wave propagation on irregular grids (Hayashi and Burns, 1999; Opršal and Zahradník, 1999), the approximation of free surface topography with the use of noncentered stencils that vary toward the free surface (Berland et al., 2007; Tarrass et al., 2011), or the design of multiscale stencils that cross boundaries of varying grid spacings (Berland et al., 2007).

In the "Theory" section, we review the standard Taylor coefficients and derive three cost functions for band-limited FD weights based on the work of Holberg (1987) and Mittet and Arntsen (1999, 2000). We then show how to implement the Remez exchange algorithm to obtain $L^{\infty}$ and $L^{2}$ optimal FD weights for the complex-valued cost functions. We demonstrate the validity of the method by contrasting a set of optimized FD operators for accurate seismic modeling against the results obtained by Liu (2014). Additionally, we will model a wave on an irregular grid. Finally, we will compare our results against the results using the method of Hicks (2002) for injecting and recording a wavefield at an arbitrary offset to an FD grid.

Supplied with this paper is a MATLAB (2017) implementation of the proposed algorithm, in the form of three stand-alone scripts that compute the optimal total, relative, and group-velocity coefficients for arbitrary derivative orders and arbitrarily sampled data. Three further scripts demonstrate how to use the algorithm to rapidly compute weights to approximate first-order derivatives on a staggered grid. Such coefficients can, for example, be used in seismic wave modeling with optimal parameters. Two final supplied files compute the Taylor coefficients for arbitrary sampling based on the algorithm of Fornberg (1998), as well as the sinc interpolation proposed by Hicks (2002).

\section{THEORY}

Consider an FD operator to approximate the $n$th derivative of a function $f(x)$ at $x=\xi$, given samples at $L$ locations $x_{l}$,

$$
\left.\frac{\mathrm{d}^{n} f(x)}{\mathrm{d} x^{n}}\right|_{x=\xi} \approx \sum_{l=1}^{L} \alpha_{l} f\left(x_{l}\right)
$$

where the real weights $\alpha_{l}$ constitute the $F D$ weights or $F D$ coefficients. We rewrite this operator for distances $\Delta_{l}=x_{l}-\xi$ and accordingly choose $f\left(x_{l}\right)=\Phi\left(\Delta_{l}\right)$ to obtain

$$
\left.\frac{\mathrm{d}^{n} \Phi(x)}{\mathrm{d} x^{n}}\right|_{x=0} \approx \sum_{l=1}^{L} \alpha_{l} \Phi\left(\Delta_{l}\right),
$$

for equivalent weights $\alpha_{l}$. The latter formulation is evaluated at $x=0$, which is a helpful simplification for the following theory.

\section{Taylor coefficients}

In the classic method, the weights $\alpha_{l}$ are obtained by replacing each $\Phi\left(\Delta_{l}\right)$ in equation 2 with a Taylor expansion around $x=0$ up to order $L-1$,

$$
\Phi\left(\Delta_{l}\right) \approx \Phi(0)+\frac{\Delta_{l} \Phi^{(1)}(0)}{1 !}+\cdots+\frac{\Delta_{l}^{L-1} \Phi^{(L-1)}(0)}{(L-1) !} .
$$

The sum of the $L$ expansions weighted with $\alpha_{l}$ must yield $\Phi^{(n)}(0)$, whereas all other terms must vanish. Following simplification, this procedure results in the following system of equations:

$$
\left[\begin{array}{llll}
\Delta_{1}^{0} & \Delta_{2}^{0} & \ldots & \Delta_{L}^{0} \\
\Delta_{1}^{1} & \Delta_{2}^{1} & \ldots & \Delta_{L}^{1} \\
\vdots & \vdots & \ddots & \vdots \\
\Delta_{1}^{L-1} & \Delta_{2}^{L-1} & \ldots & \Delta_{L}^{L-1}
\end{array}\right]\left[\begin{array}{l}
\alpha_{1} \\
\alpha_{2} \\
\vdots \\
\alpha_{L}
\end{array}\right]=n ! \mathbf{e}_{\mathbf{n}+\mathbf{1}},
$$

where $\mathbf{e}_{\mathbf{n}}$ is a unit vector with value 1 only on the $n$th row. We then invert the $L \times L$ system of equations to obtain $\alpha_{l}$, for which efficient methods exist (Fornberg, 1988; Khan and Ohba, 2000; Bowen and Smith, 2005). The Taylor coefficients give the exact interpolation or differentiation of polynomials of the degree that the Taylor polynomials were expanded to,

$$
f(x)=a_{0}+a_{1} x+a_{2} x^{2}+\ldots+a_{L-1} x^{L-1} .
$$

\section{Band-limited coefficients}

\section{Comparison to Taylor coefficients}

We now choose $\Phi\left(\Delta_{l}, k\right)=e^{ \pm i k \Delta_{l}}$ as a complex-valued plane wave with wavenumber $k=\omega / c$, for angular frequency $\omega$ and phase velocity $c$. Upon substitution of $\Phi\left(\Delta_{l}, k\right)$ in equation 2 , we obtain

$$
( \pm i k)^{n} \approx \sum_{l=1}^{L} \alpha_{l} e^{ \pm i k \Delta_{l}}
$$

Note that we could rewrite the plane-wave function with a Taylor series around $k=0$ as

$$
\Phi(\Delta, k)=e^{ \pm i k \Delta}=1+\frac{ \pm i k}{1} \Delta+\frac{( \pm i k)^{2}}{2 !} \Delta^{2}+\ldots
$$

The Taylor series of equation 3 can now be recognized as a truncated version of equation 7. Therefore, the Taylor coefficients apply precisely in close vicinity of $k=0 \mathrm{rad} / \mathrm{m}$, but deteriorate with increasing $k$ by not incorporating higher order terms. The band-limited coefficients, conversely, should apply for a larger range of $k$, at the expense of allowing errors over the entire wavenumber range, including errors at $k=0 \mathrm{rad} / \mathrm{m}$.

\section{A total cost function}

We will now discuss the design of band-limited plane-wave coefficients. We express the approximation error of equation 6 as 


$$
E_{n}(k)=W(k)\left[( \pm i k)^{n}-\sum_{l=1}^{L} \alpha_{l} e^{ \pm i k \Delta_{l}}\right],
$$

where $W$ is a weight function chosen as

$$
W(k)= \begin{cases}1 & k \in \text { range of interest } \\ 0 & k \in \text { irrelevant range }\end{cases}
$$

Assume that we choose the range of interest to be the interval $\left[0, K_{c}\right] \mathrm{rad} / \mathrm{m}$ up to some chosen critical wavenumber $K_{c}$. We then write the total error as

$$
E_{n}(k)= \begin{cases}( \pm i k)^{n}-\sum_{l=1}^{L} \alpha_{l} e^{ \pm i k \Delta_{l}} & k \in\left[0, K_{c}\right] \\ 0 & \text { otherwise }\end{cases}
$$

\section{Relative and group-velocity cost function}

The target function $( \pm i k)^{n}$ increases with $k$ for $n>0$, whereas the total error weighs all errors equally along $k$. We can more carefully weigh the errors by pursuing a relative cost function that penalizes errors relative to the target function $( \pm i k)^{n}$, such that errors at low wavenumbers are penalized more than errors at high wavenumbers. We, therefore, choose a new weight function to be used in conjunction with equation 8 :

$$
W(k)= \begin{cases}\frac{1}{( \pm i k)^{n}} & k \in\left[0, K_{c}\right] \\ 0 & \text { otherwise }\end{cases}
$$

yielding the relative cost function

$$
E_{r n}(k)= \begin{cases}1-\sum_{l=1}^{L} \alpha_{l} \frac{e^{ \pm i k \Delta_{l}}}{( \pm i k)^{n}} & k \in\left[0, K_{c}\right] \\ 0 & \text { otherwise }\end{cases}
$$

Note that the fraction $e^{ \pm i k \Delta_{l}} /( \pm i k)^{n}$ is singular at $k=0$, but the summation with all $\alpha_{l}$ can be constrained to a finite value by substituting equation 7 into the exponential of equation 12 ,

$$
\sum_{l=1}^{L} \alpha_{l} \frac{e^{ \pm i k \Delta_{l}}}{( \pm i k)^{n}}=\sum_{l=1}^{L} \alpha_{l} \frac{1+\frac{ \pm i k}{1} \Delta_{l}+\frac{( \pm i k)^{2}}{2 !} \Delta_{l}^{2}+\ldots}{( \pm i k)^{n}}
$$

Only the terms in the numerator with $( \pm i k)^{m}$ for $m<n$ are singularities, whereas terms with $m>n$ equal 0 for $k=0$. Thus, if we desire that equation 13 equals, e.g., 1 at $k=0 \mathrm{rad} / \mathrm{m}$, the following constraints must be satisfied:

$$
\sum_{l=1}^{L} \alpha_{l} \Delta_{l}^{m}= \begin{cases}0 & \text { for } 0 \leq m<n \\ n ! & \text { for } m=n\end{cases}
$$

These constraints are the constraints of equation 4 up to the derivative of order $n$.

A final cost function is the group-velocity error (Holberg, 1987; Mittet and Arntsen, 1999, 2000), defined as $k E_{r n}^{\prime}(k)+E_{r n}(k)$, resulting in

$$
E_{g n}(k)= \begin{cases}1-\sum_{l=1}^{L} \alpha_{l} \frac{1-n \pm i k \Delta_{l}}{( \pm i k)^{n}} e^{ \pm i k \Delta_{l}} & k \in\left[0, K_{c}\right] \\ 0 & \text { otherwise }\end{cases}
$$

The constraints from equation 14 also apply to ensure existence of a solution at $k=0 \mathrm{rad} / \mathrm{m}$ for the group-velocity error. This cost function favors FD operators with a nearly flat relative error after an initial deviation at $k=0 \mathrm{rad} / \mathrm{m}$. In Appendix $\mathrm{B}$, we show that the relative and group-velocity cost functions describe the error in the phase and group velocity, respectively, when FD operators are used for seismic modeling. Furthermore, we show that the maximum relative error is bounded by the maximum group-velocity error, such that a small group-velocity error ensures a small relative error also.

\section{Solving the $L^{\infty}$ optimization problem}

\section{A complex-valued error}

Although the target functions $( \pm i k)^{n}$ are either real or imaginary, the FD approximation can be a combination of both. The situation is simpler when the samples are chosen symmetrically; that is, if for every sample at $\Delta_{l}$, there is another sample at $\Delta_{m}=-\Delta_{l}$. In that symmetric case, we assume that for each tuple $\left\{\Delta_{l}, \alpha_{l}\right\}$, there is a complementary pair $\left\{\Delta_{m}=-\Delta_{l}, \alpha_{m}=(-1)^{n} \alpha_{l}\right\}$. The summed pair $\alpha_{l} e^{i k \Delta_{l}}+\alpha_{m} e^{i k \Delta_{m}}=\alpha_{l}\left(e^{i k \Delta_{l}}+(-1)^{n} e^{-i k \Delta_{l}}\right)$ equals $2 \alpha_{l} \cos \left(k \Delta_{l}\right)$ for even $n$ and $2 i \alpha_{l} \sin (k \Delta l)$ for odd $n$. Hence, the error would be fully real for even derivatives and fully imaginary for odd derivatives. In the general case, however, the error of the FD operator may be a combination of a real and imaginary component.

For the $L^{\infty}$ optimization, we aim to obtain the coefficients $\alpha_{l}$ for which the FD operator has a minimal absolute error for the largest wavenumber range, which is a minimax optimization problem,

$$
\underset{\alpha_{l}}{\arg \min }\left\|E_{n}(k)\right\|_{\infty}=\underset{\alpha_{l}}{\arg \min } \max _{k \in\left[0, K_{c}\right]}\left|E_{n}(k)\right| .
$$

The solution to such problems is equiripple. This means that the maximum absolute error is reached exactly, say, $m$ times:

$$
\left|E\left(k_{j}\right)\right|=\left|E\left(k_{j+1}\right)\right|, \quad \text { for all } 1 \leq j<m .
$$

Noting that the complex-valued error can be written as the product of its magnitude and phase, $E(k)=|E(k)| e^{i \theta(k)}$, we thus realize that it is only the phase $e^{i \theta\left(k_{j}\right)}$ that varies between the maxima. We can obtain the $m$ maximum wavenumbers, phases, and the maximum error $\|E(k)\|_{\infty}$ with the Remez exchange algorithm (Remez, 1934; McClellan et al., 1973), extended to the complex domain (Preuss, 1987; Schulist, 1990; Alkhairy, 1994). We illustrate this for the total error, but it generalizes in a straightforward way also to the relative and group-velocity cost functions.

\section{The Remez exchange algorithm}

Consider the total error of equation 10 for maxima at $k_{j}$,

$$
\left|E_{n}\left(k_{j}\right)\right| e^{ \pm i \theta\left(k_{j}\right)}+\sum_{l=1}^{L} \alpha_{l} e^{ \pm i k_{j} \Delta_{l}}=\left( \pm i k_{j}\right)^{n}
$$

which can be written as a matrix-vector system

$$
\mathbf{D h}=\mathbf{d},
$$

with the desired real-valued vector of FD coefficients and the total absolute error 


$$
\mathbf{h}=\left[\begin{array}{l}
\alpha_{1} \\
\alpha_{2} \\
\vdots \\
\alpha_{L} \\
\left|E_{n}\left(k_{1}\right)\right|
\end{array}\right]
$$

The matrix D contains all sampling locations and error phases, whereas the vector $\mathbf{d}$ contains the target function,

$$
\mathbf{D}=\left[\begin{array}{llll}
e^{+i k_{1} \Delta_{1}} & \ldots & e^{+i k_{1} \Delta_{L}} & e^{+i \theta_{1}} \\
e^{-i k_{1} \Delta_{1}} & \ldots & e^{-i k_{1} \Delta_{L}} & e^{-i \theta_{1}} \\
e^{+i k_{2} \Delta_{1}} & \ldots & e^{+i k_{2} \Delta_{L}} & e^{+i \theta_{2}} \\
e^{-i k_{2} \Delta_{1}} & \ldots & e^{-i k_{2} \Delta_{L}} & e^{-i \theta_{2}} \\
\vdots & \ddots & \vdots & \vdots \\
e^{+i k_{m} \Delta_{1}} & \ldots & e^{+i k_{m} \Delta_{L}} & e^{+i \theta_{m}} \\
e^{-i k_{m} \Delta_{1}} & \ldots & e^{-i k_{m} \Delta_{L}} & e^{-i \theta_{m}}
\end{array}\right], \mathbf{d}=\left[\begin{array}{l}
\left(+i k_{1}\right)^{n} \\
\left(-i k_{1}\right)^{n} \\
\left(+i k_{2}\right)^{n} \\
\left(-i k_{2}\right)^{n} \\
\vdots \\
\left(+i k_{m}\right)^{n} \\
\left(-i k_{m}\right)^{n}
\end{array}\right] .
$$

Note that at the last local maximum $k_{m}=K_{c}$ by construction. The Remez exchange algorithm solves equation 19 by iteratively obtaining the optimal $\alpha_{l}$ using an estimate of the local maxima $\left\{k_{j}, \theta_{j}\right\}$; and then searching the maxima $\left\{k_{j}, \theta_{j}\right\}$ that result from the obtained $\alpha_{l}$. In other words, we iteratively estimate $\mathbf{h}$ from $\mathbf{D}$ and $\mathbf{D}$ from $\mathbf{h}$ until convergence. The number of maxima $m$ is

$$
m= \begin{cases}\left\lceil\frac{L+1}{2}\right\rceil & \text { for the total error, } \\ \left\lceil\frac{L+1-n}{2}\right\rceil & \text { for the relative and group-velocity error, }\end{cases}
$$

where $\lceil\cdot\rceil$ is the ceiling operator that rounds to the nearest integer above. Further details on how this number is obtained and a discussion about the Remez exchange algorithm can be found in Appendix A. The complex-valued multiple exchange algorithm then proceeds as follows:

Step 0) Initialize: Choose the order of differentiation $n$, the extremal wavenumber $K_{c}$, the number of maxima $m$, a linearly spaced set of corresponding wavenumbers, $k_{j}=(j-1) K_{c} /(m-1)$, and an oscillating set of phases, $\theta_{j}=(j-1) \pi$. Choose the cost function (equations 10/12/15), which prescribes the structure of the matrices in equation 19.

Step 1) Solve: Invert equation 19 to obtain the FD weights (using, e.g., MATLAB's backslash operator),

$$
\mathbf{h}=\mathbf{D}^{-1} \mathbf{d} .
$$

Step 2) Exchange: Compute the error $E(k)$ over a dense grid of wavenumbers, using the weights obtained from the matrix inversion. Obtain a new set of $m$ maximum wavenumbers and phases $\left\{k_{j}, \theta_{j}\right\}$ from the maxima of $|E(k)|$.

Step 3) Evaluate: Compute $\|E\|_{\infty}=\max _{1 \leq j \leq m}\left|E\left(k_{j}\right)\right|$. Stop iterations when no further progress is made, e.g.,

$$
\frac{\left|\|E\|_{\infty}-\left\|E^{\text {previous iteration }}\right\|_{\infty}\right|}{\|E\|_{\infty}} \leq 10^{-10} \Rightarrow \text { terminate. }
$$

Otherwise, go to step 1 and iterate again.

\section{Solving the $L^{2}$ optimization problem}

The least-squares optimization problem is formulated as

$$
\underset{\alpha_{l}}{\arg \min }\|E(k)\|_{2}, \quad \forall k \in\left[0, K_{c}\right] .
$$

Solutions to this optimization problem tend to trade off small errors at low wavenumbers at the expense of large errors at larger wavenumbers. This optimization problem is straightforward to solve with steps 0 and 1 of the Remez exchange algorithm, using a large $m$ (e.g., $m=10^{6}$ ) such that equation 19 becomes overdetermined and considers all wavenumbers $k$. A single matrix inversion then provides the $L^{2}$-optimal weights. Note that it is beneficial to obtain the $L^{2}$-optimal solution to accelerate the $L^{\infty}$ optimization also: the maxima of the $L^{2}$ error function lie close to those of the $L^{\infty}$ solution and thus provide good initialization for $k_{m}$ and $\theta_{m}$ in the Remez exchange algorithm.

\section{Output example}

In Figure 1, we compare four designed "optimal" symmetric differentiators to the Taylor coefficients as an example of the different traits due to the cost functions and optimality conditions. The coefficients are generated by running the following MATLAB commands with the provided code:

- $\left\|E_{r 1}\right\|_{2}$-optimality: FD_rel $(-6.5: 6.5,1,2.07,1 \mathrm{e} 3)$,

- $\left\|E_{g 1}\right\|_{2}$-optimality: FD_gv $(-6.5: 6.5,1,1.715,1 \mathrm{e})$,

- $\left\|E_{r 1}\right\|_{\infty}$-optimality: FD_rel $(-6.5: 6.5,1,2.1,1)$,

- $\left\|E_{g 1}\right\|_{\infty}$-optimality: FD_gv $(-6.5: 6.5,1,1.747,1)$,

where the first argument specifies the sampling points $\left(\Delta_{l}\right)$, the second argument specifies the order of differentiation $(n=1)$, the third argument specifies the critical wavenumber $\left(K_{c}\right)$ up to which the operator is optimized, and the final provided argument specifies the number of expected maxima $m$ beyond that given in equation 22 . The least-squares solutions follow from oversampling the number of maxima. The equiripple solution of the symmetric first derivative also requires one more maximum than expected from equation 22 , as explained in Appendix A. Note how, for this fixed maximum error, the equiripple operators cover an approximately $3 \%$ larger wavenumber range for an identical maximum error as the least-squares operators. Furthermore, we may observe that the operators optimized for the relative cost function cover a larger bandwidth than the group-velocity optimal operators. However, we may observe that the group-velocity optimal weights display a nearly flat relative-error response after an initial deviation. Finally, for any given maximum error, the optimal FD weights cover a larger bandwidth than the Taylor weights.

Additionally, consider Table 1, where we compare the output for an "asymmetric" FD interpolation against the Taylor coefficients and the results of Hicks (2002). The coefficients can be generated with the MATLAB file using the following commands:

- $\quad\left\|E_{r 0}\right\|_{2}$-optimality: FD_rel $(-2.3: 1.7,0,1,1 \mathrm{e} 3)$,

- $\quad\left\|E_{g 0}\right\|_{2}$-optimality: FD_gv $(-2.3: 1.7,0,1,1 \mathrm{e})$,

- $\left\|E_{r 0}\right\|_{\infty}$-optimality: FD_rel $(-2.3: 1.7,0,1)$,

- $\left\|E_{g 0}\right\|_{\infty}$-optimality: FD_gv $(-2.3: 1.7,0,1)$,

where the second argument to the script now signifies interpolation $(n=0)$ and the third argument fixes the wavenumber range, $K_{c}=1$. The second set of coefficients in Table 1 is obtained by setting the third argument to $K_{c}=2$. Note that the coefficients designed with 
the optimization procedure follow the general trend of the Taylor coefficients closely. Indeed, the output of the optimizations will tend toward the Taylor coefficients for $K_{c} \rightarrow 0$.

\section{ACCURATE FD MODELING OF WAVE PROPAGATION}

We apply the methodology of the previous section to three FD modeling problems. In the first two examples, we design first- and second-order FD differentiators to simulate seismic waves with minimal dispersion on a regular and irregular grid. In the third example, we inject and record a wave offset with respect to the grid using optimized FD interpolators.

\section{Uniform, 1D first-order simulation}

We model the 1D acoustic wave equation as a system of first-order partial differential equations governing pressure $p$ and particle velocity $v$. Under the assumption of a homogeneous unit density, the physical relations are

$$
\begin{aligned}
& \frac{\partial p(x, t)}{\partial t}=-c^{2}(x)\left(\frac{\partial v(x, t)}{\partial x}+\delta\left(x_{s}-x\right) q(t)\right), \\
& \frac{\partial v(x, t)}{\partial t}=-\frac{\partial p(x, t)}{\partial x}
\end{aligned}
$$

for a source located at $x_{s}$ and source-time function $q(t)$. We use the notation $p(i \Delta x, n \Delta t)=p_{i}^{n}$ to denote the quantities located at a regular distance $\Delta x$ in space and $\Delta t$ in time. We then solve the system numerically following the staggered-grid leap-frog system devised by Virieux (1986),

$$
\frac{p_{i+\frac{1}{2}}^{n+\frac{1}{2}}-p_{i+\frac{1}{2}}^{n-\frac{1}{2}}}{\Delta t}=-c_{i}^{2} \sum_{l=1}^{L} \frac{\alpha_{l}}{\Delta x}\left(v_{i+l}^{n}-v_{i-(l-1)}^{n}\right)-c_{i}^{2} \bar{\delta}_{i_{s}+\frac{1}{2}-i} q^{n},
$$

$$
\frac{v_{i}^{n+1}-v_{i}^{n}}{\Delta t}=\sum_{l=1}^{L} \frac{\alpha_{l}}{\Delta x}\left(p_{i+l-\frac{1}{2}}^{n+\frac{1}{2}}-p_{i-l+\frac{1}{2}}^{n+\frac{1}{2}}\right)
$$

The point source $\delta_{i_{s}+1 / 2-i}=1 / \Delta x$ when $i_{s}+1 / 2-i=0$ and zero otherwise. As a boundary condition, we impose a free surface on both sides of the grid through the method of imaging by modeling the pressure as an odd function over the boundary and modeling the particle velocity as an even function over the boundary (Levander, 1988; Crase, 1990). The FD coefficients $\alpha_{l}$ are designed for $\Delta x=1$ $\mathrm{m}$, such that they can be reused for arbitrary $\Delta x$.

\section{Homogeneous medium example}

We will solve the system of equations 28 and 29 numerically in a homogeneous example only. We limit ourselves to this case because (1) a homogeneous domain produces the most dispersion and (2) the accuracy gains of the FD operator may be offset by the inaccuracy of implemented medium interfaces. First, because a wave in a homogeneous model occupies a constant wavenumber region, it accumulates the maximum amount of dispersion along its path. In a heterogeneous domain, the wave could accumulate differing and potentially offsetting dispersion errors. The second point is that medium interfaces, even when properly band-limited, only deliver up to sixthorder accuracy (Mittet, 2017). A homogeneous model is thus the best test case to isolate the errors of the FD operator.

We choose a wave velocity $c=2000 \mathrm{~m} / \mathrm{s}$, for 301 nodes with $\Delta x=10 \mathrm{~m}$ from 0 up to $3000 \mathrm{~m}$. We choose $\Delta t=1 \mathrm{~ms}$ and model for $0.8 \mathrm{~s}$. We use a Ricker wavelet with a $40 \mathrm{~Hz}$ peak frequency, yielding five grid points per dominant wavelength. The source is injected at $755 \mathrm{~m}$ and recorded at $1505 \mathrm{~m}$ (i.e., coincident with the staggered grid pressure nodes). We test the performance of an FD operator of half-length $L=19$ to compare our results with those from Liu (2014, their Table 10). The latter coefficients are least-squares optimal FD coefficients up to a maximum relative error of $10^{-4}$ at a maximum wavenumber of $K_{c}=2.9$. The coefficients designed by Liu (2014) have zero error at zero wavenumbers, unlike the coefficients proposed
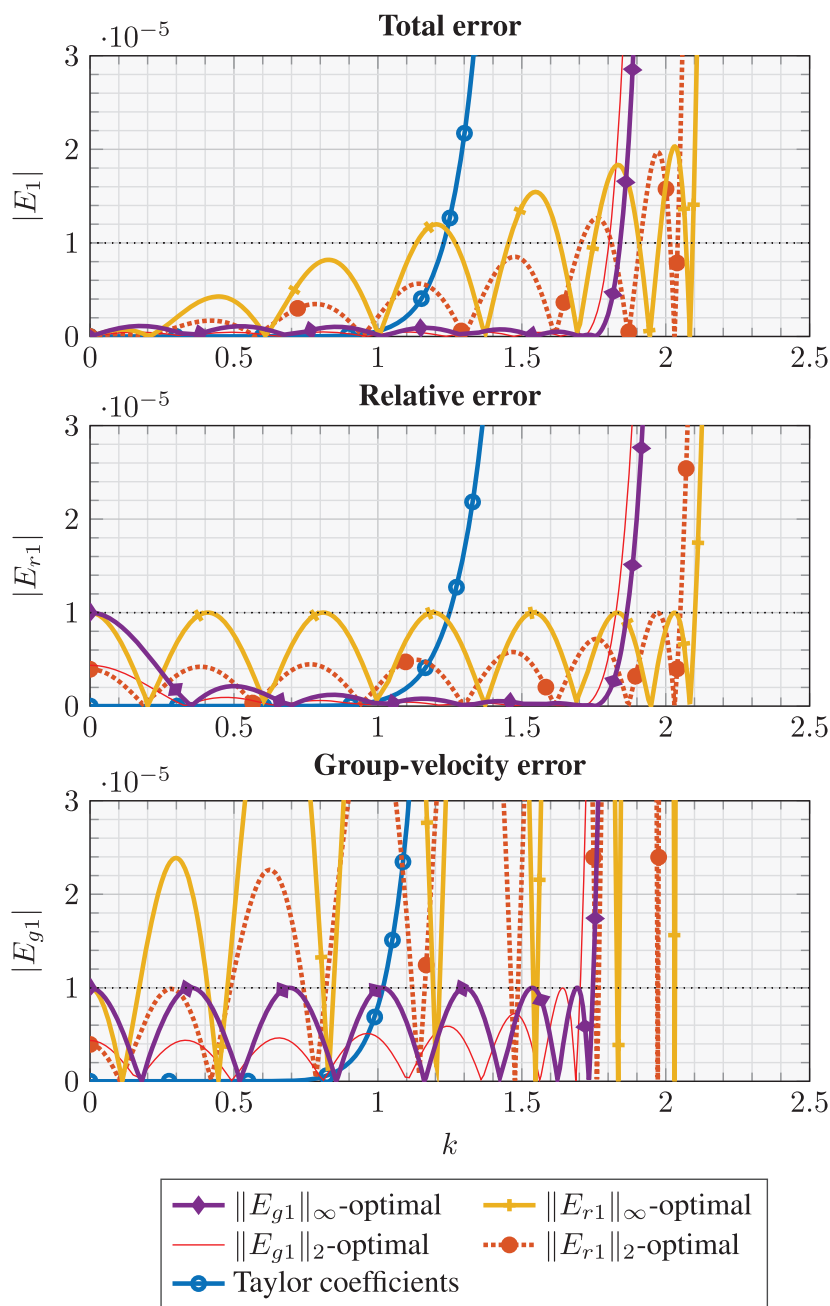

Figure 1. The error of five FD operators to approximate a first-order derivative, sampled symmetrically at $\Delta_{l}=\{-6.5,-5.5, \ldots, 6.5\}$. The Taylor coefficients cover a small bandwidth with nearly no error. Conversely, the $L^{2}$-optimal and $L^{\infty}$-optimal operators cover a larger bandwidth, at the expense of oscillating errors. The $L^{\infty}$-optimal weights cover a larger bandwidth than the $L^{2}$-optimal weights for any maximum error. The group-velocity optimal weights create a "flat" relative error behavior. 
in this paper. We design 12 sets of coefficients for comparison. The first six have the same maximum error for their respective cost functions, for the least-squares and equiripple solution:

Table 1. Four different FD operators to interpolate five samples. The coefficients due to Hicks (2002) are obtained using $b=6.31$ in his method. The coefficients are rounded to four decimals.

Method

$\Delta_{l}$

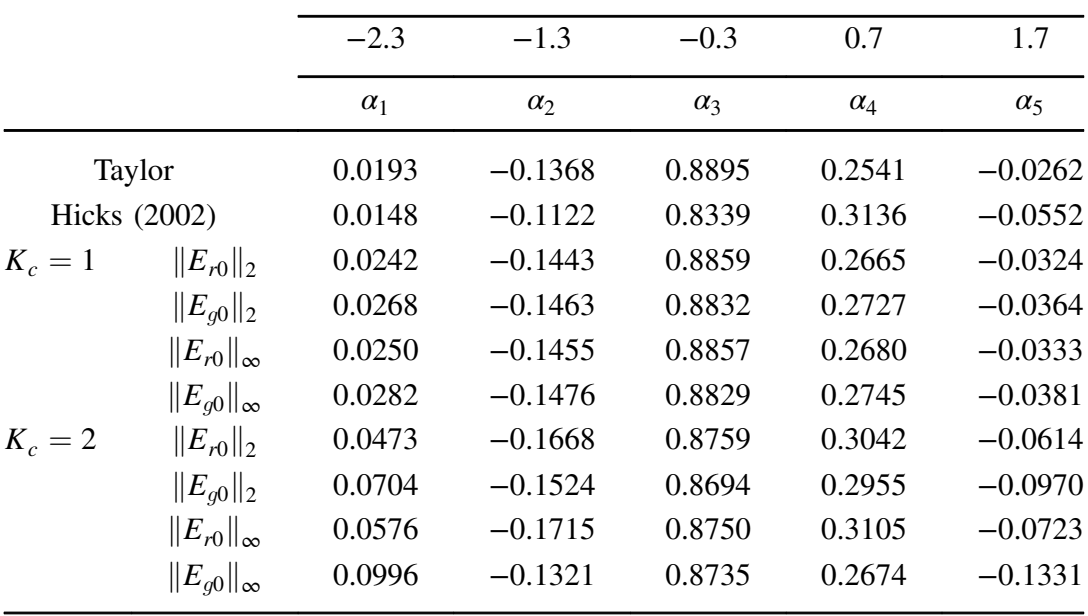

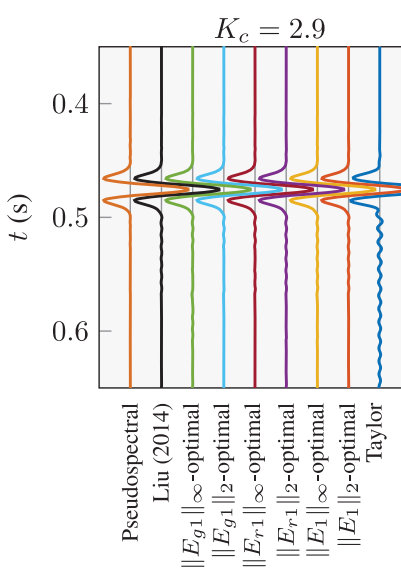

Error $\times 20$

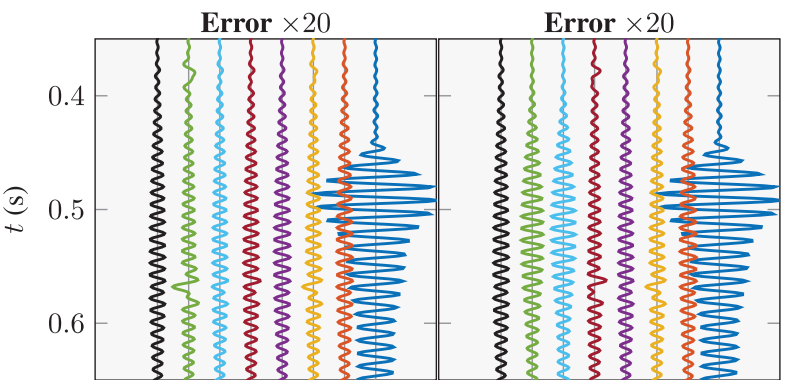

Figure 2. The outcome of 14 FD simulations with varying operators. The traces are compared against a simulation with the pseudospectral method, which is considered to be the reference solution. Apart from the solution due to the Taylor series, the results are nearly indistinguishable. The solution due to the $\left\|E_{r 1}\right\|_{\infty}=10^{-4}$-optimal operator (in the bottom-right plot) has the smallest error, which gives $10 \%$ smaller fluctuations than the least-squares optimal solution from either $\left\|E_{r 1}\right\|_{2}$ or Liu (2014).

$$
\max \text { error }\left\{\begin{array}{l}
\left\|E_{1}(k)\right\|_{2, \infty} \\
\left\|E_{r 1}(k)\right\|_{2, \infty} \\
\left\|E_{g 1}(k)\right\|_{2, \infty}
\end{array}=10^{-4}\right.
$$

The second set of six FD operators is designed to be optimal up to the same critical wavenumber $K_{c}=2.9$,

$$
\text { range }\left\{\begin{array}{l}
\left\|E_{1}(k)\right\|_{2, \infty}, \\
\left\|E_{r 1}(k)\right\|_{2, \infty}, k \in[0,2.9] . \\
\left\|E_{g 1}(k)\right\|_{2, \infty},
\end{array}\right.
$$

The computed FD weights can be found with the online supplementary files or can be created by running the MATLAB commands table2.m and table $3 . \mathrm{m}$. A reference solution is computed using the staggered pseudospectral scheme from Özdenvar and McMechan (1996) to obtain the maximum possible accuracy on this homogeneous simulation. We use the time-dispersion removal procedure from Koene et al. (2018) to remove the effect of time dispersion from the recorded traces. The remaining dispersion error due to the FD operator then consists of trailing oscillations behind the peak arrival of the wavelet (Dablain, 1986). The optimal FD operator should reduce this ringing as much as possible. The resulting simulations may be reproduced with the provided MATLAB script example_uniform_grid_1D.m.

Figure 2 shows the time series resulting from these simulations. Each simulation takes approximately $15 \mathrm{~s}$ on a single-node desktop $\mathrm{PC}$, whereas the estimation of the coefficients only takes between 0.01 and $0.2 \mathrm{~s}$. First, we note that the simulation using the Taylor weights generates significant "ringing" behind the peak of the wavelet for the $40 \mathrm{~Hz}$ Ricker wavelet: a clear sign of spatial dispersion. In comparison, the pseudospectral method is nearly free of any ringing. This means that infinitely large stencils can suppress the dispersion satisfactorily (Fornberg, 1990), and our stencils should approximate this effect. A second observation is that all optimal FD coefficients provide significantly fewer dispersion artifacts, minimizing the ringing roughly equally well. The smallest dispersion oscillations are obtained with the $\left\|E_{r 1}\right\|_{\infty}=10^{-4}$-optimal coefficients, with oscillatory extrema $10 \%$ smaller than that obtained with Liu's coefficients. However, note also that the equiripple solutions contain small leading and trailing artifacts (visible at $t=0.375$ and $t=0.56 \mathrm{~s}$ ). The origin of these artifacts probably lies in the large allowed relative error at low wavenumbers. If such artifacts are deemed to be undesired, the second-best and artifact-free results are obtained with the $\left\|E_{r 1}\right\|_{2}$-optimal weights or those by Liu (2014), which produce virtually identical results.

\section{Irregular 1D second-order simulation}

We will now model the acoustic wave equation using a secondorder partial differential equation:

$$
\frac{\partial^{2} p(x, t)}{\partial t^{2}}=c^{2}(x)\left(\frac{\partial^{2} p(x, t)}{\partial t^{2}}+\delta\left(x-x_{s}\right) q(t)\right) .
$$


We will assume that the wavefield is distributed irregularly in space but regularly in time. We will use the index notation of the previous section, but with the meaning that $p_{i}^{n}=p\left(x_{i}, n \Delta t\right)$, where $x_{i}$ is sorted in ascending order. The irregular spacing can, for example, allow for large spacing at high velocities and small spacing for low velocities or rapidly fluctuating medium properties. The equation is solved numerically as

$$
\frac{p_{i}^{n+1}-2 p_{i}^{n}+p_{i}^{n-1}}{\Delta t^{2}}=c_{i}^{2}\left(\sum_{l=-L}^{L} \alpha_{i+l} p_{i+l}^{n}\right)+c_{i}^{2} \delta_{i_{s}-i} q^{n} .
$$

The point source $\delta_{i_{s}-i}=2 /\left(x_{i_{s}+1}-x_{i_{s}-1}\right)$ for $i_{s}-i=0$ uses the reciprocal of the average step size around the source injection node, and it is zero otherwise. Because each node is arbitrarily positioned within the irregular grid, we require as many FD operators as nodes $i$. We create a free surface on the edges by setting the outer nodes to zero, $p_{1}=p_{M}=0$. We will use a one-sided FD grid at the boundaries. The authors are not aware of other papers in the literature where high-order FD wave simulations are carried out on truly irregular grids, and additionally with noncentered stencils around the free surface, although mimetic FD operators may provide an attractive alternative approach to such problems (Shragge and Tapley, 2017). The advantage of using a noncentered grid at the free surface is that nonphysical ghost nodes do not have to be invoked in the computation of the derivatives.

\section{Homogeneous medium example}

We again focus on a homogeneous example as the worst-case scenario for FD operators. We choose a wave velocity $c=2000 \mathrm{~m} / \mathrm{s}$, for $M=301$ nodes. The node spacing follows a cosine distribution and a random draw:

$$
x_{i}=\frac{\cos (\theta)-\cos \left(\theta+\frac{i-1}{M-1} 2 \theta\right)}{2 \cos (\theta)} \times 3000+\mathcal{N}_{i}(\mu=0, \sigma=1) \text {, }
$$

for $\theta=\pi / 6$, and where $\mathcal{N}_{i}(\mu=0, \sigma=1)$ draws a random number for each $i$ from a normal Gaussian distribution. In the MATLAB script, we use a fixed random seed to ensure the reproducibility of the result. We choose $x_{M}=3000-x_{1}$, such that the domain is exactly $3000 \mathrm{~m}$ long, producing a multiple after $3 \mathrm{~s}$. The smallest spatial distance is $2 \mathrm{~m}$ toward the border of the domain, whereas the largest spatial distance is $16 \mathrm{~m}$ in the center of the domain. See Figure $3 \mathrm{a}$ for a magnified plot with the node positions up to $500 \mathrm{~m}$. We inject a $30 \mathrm{~Hz}$ Ricker wavelet at the 75th node $(622 \mathrm{~m})$ and record it at the 150th node $(1489 \mathrm{~m})$. We use $\Delta t=1 \mathrm{~ms}$ and model up to $4.7 \mathrm{~s}$. We use the time-dispersion removal procedure from Koene et al. (2018) to remove all effects of time dispersion. We use the stencil half-length $L=$ 6 (using 13 nodes), $L=14$ (using 29 nodes), and $L=22$ (using 43 nodes) to compare results for different stencil lengths. In Figure $3 b$, we show the stencils for $L=6$ for the grid nodes up to $500 \mathrm{~m}$.

The one-sided boundary is a challenge to implement because various operators are inherently unstable at the boundary (see also Shragge and Tapley [2017] concerning the long-term stability of one-sided operators). In particular, the designed Taylor coefficients and the equiripple operators are unconditionally unstable at the boundary - the instability even occurs when using a regular, rather than an irregular, grid, and for any value of the time-step $\Delta t$. The leastsquares total-error and group-velocity optimal operators are stable but inaccurate at the boundaries. These instabilities and inaccuracies may be alleviated by low-pass filtering during the simulation (Berland et al., 2007; Tarrass et al., 2011). However, we found that we could obtain a stable and accurate implementation without filtering by using the relative error optimal least-squares operator. The FD operators are optimized up to $K_{c}=\pi /(1.25 \overline{\Delta x})$ in the center and up to $K_{c}=$ $\pi / \overline{\Delta x}$ at the boundaries, where $\overline{\Delta x}$ is the median spatial step size in the range of the stencil. That means that at the boundaries, we cover the entire signal up to the Nyquist bandwidth because the wave's polarity flip (induced by the free-surface boundary) may contain energy up to the Nyquist frequency. Conversely, the rest of the grid is optimized over the required bandwidth only.

It takes an average of $20 \mathrm{~s}$ to run the simulations, whereas it takes less than $0.1 \mathrm{~s}$ to obtain all 301 stencils in each of the three cases. We demonstrate that the method is stable and accurate in the long term, and we show the entire $p(x, t)$ solution in Figure $4 a$. The wave moves at a constant speed even along the boundaries and produces a perfect multiple after $3.0 \mathrm{~s}$. We can thus compare traces from $t \in$ $(0,1.4) \mathrm{s}$ and $t \in(3,4.4) \mathrm{s}$ (see Figure $4 \mathrm{~b})$. The later arriving trace for the $L=6$ operator is ringy and hence dispersed, which is also visible in the large amplitudes of the difference plot. When we increase the stencil length to $L=14$ and $L=22$, the difference plot shows significantly smaller differences; hence, the dispersion errors are significantly smaller. We can note, however, that the accuracy improvement from $L=14$ to $L=22$ is only small. Thus, we were able to (1) model waves accurately on irregular grids and (2) use noncentered grids to model a free surface accurately, sidestepping the need to invoke ghost nodes. This simulation can be reproduced with the script variable_gridspacing_1D.m.

\section{$2 \mathrm{D}$ recording and injecting a point-source at an arbitrary location}

To record wavefields on the FD grids, we typically save the value at a certain node over time. However, when the recording position does not coincide with the grid, we must interpolate the data using, e.g., FD operators. To do this in two dimensions, for example, we

a)

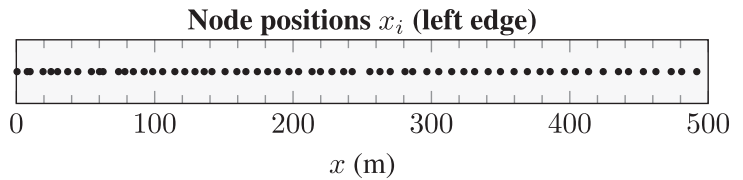

b)

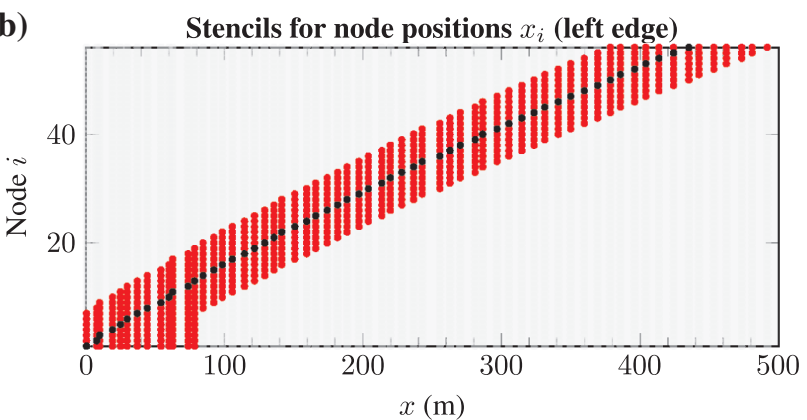

Figure 3. (a) A plot of the irregular grid $x_{i}$, zoomed in on the left edge. Every black dot represents a location of $x_{i}$. Note the irregularity of the nodes. (b) The corresponding stencils for each node (in black) are spanned by the red dots in the horizontal direction. The stencil is one-sided on the left boundary and centered with an equal number of nodes on both sides elsewhere. 
identify a block of $L \times M$ samples $p\left(i_{l}, j_{m}\right)$, in which an arbitrary point $p(\xi, \zeta)$ falls. Then, we perform $L$ interpolations along all $L$ columns, to obtain $p\left(\xi, j_{m}\right)$. Finally, we perform a single interpolation along the $M$ number of $p\left(\xi, j_{m}\right)$ to obtain $p(\xi, \zeta)$. This equals a double summation:

$$
p(\xi, \zeta, t)=\sum_{l=1}^{L} \sum_{m=1}^{M} \alpha_{l} \beta_{m} p\left(i_{l}, j_{m}, t\right) .
$$

For a point-source injection that does not coincide with the grid, a reciprocal relationship holds, with the identical interpolation weights. We interpolate the point source onto an array of points on the grid. The locations surround the desired source location, and the interpolation is carried out with the FD weights (see Figure 5). Thus,

$$
\delta_{i_{l}, j_{m}}=\sum_{l=1}^{L} \sum_{m=1}^{M} \alpha_{l} \beta_{m} \delta_{i_{l}-i_{0}, j_{m}-j_{0}}=\frac{\alpha_{l} \beta_{m}}{\Delta x \Delta z} .
$$

\section{$2 D$ wavefield recording and injection example}

In this example, we use a homogeneous model with a propagation velocity $c=1500 \mathrm{~m} / \mathrm{s}$ with grid spacing $\Delta x=\Delta z=10 \mathrm{~m}$,

a)

1D wave propagation in space-time domain

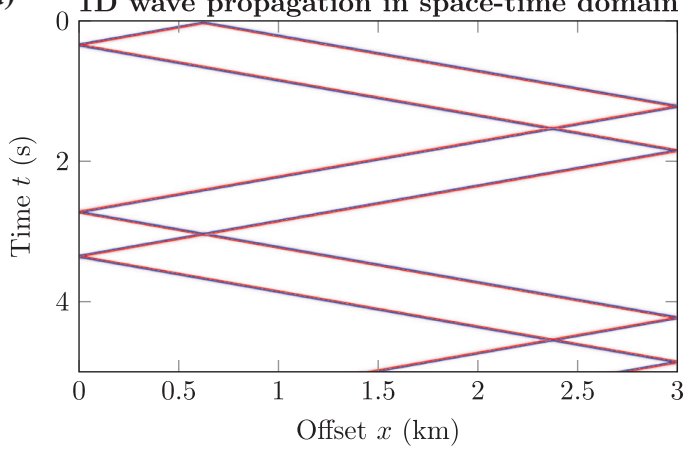

b)

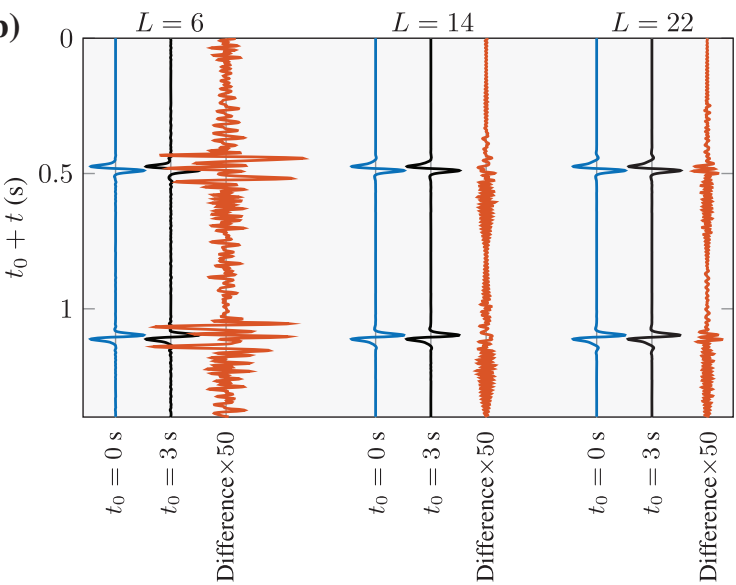

Figure 4. Results for three acoustic FD simulations on an irregular grid. (a) A figure of the wave propagation for $4.7 \mathrm{~s}$ in the $(x, t)$ plane on the irregularly spaced grid. The stencil used a half-length of $L=22$. Note how the wave pattern repeats after $3 \mathrm{~s}$. (b) A comparison of three acoustic FD simulations on an irregular grid. We compare traces recorded on two intervals, from $t=0 \mathrm{~s}$ to $t=1.4 \mathrm{~s}$ and from $t=3 \mathrm{~s}$ to $t=4.4 \mathrm{~s}$. The $L$ in the title indicates the half-length of the operator. The difference plot, indicative of the amount of dispersion, reduces for increasing $L$. for 251 nodes in the $x$-direction and 100 nodes in the $z$-direction. The source-time function is a Ricker wavelet with $20 \mathrm{~Hz}$ central frequency. We use the standard Taylor coefficients in the differentiator of equation 28, extended to two dimensions, for operator halfwidth $L=7$. The system is solved in two dimensions following the 2D staggered-grid (Figure 6) devised by Virieux (1986).

We create a reference simulation where a wavefield is injected and recorded on the grid, using a source at $(x, z)=(1200,190) \mathrm{m}$ and a receiver at $(x, z)=(1700,690) \mathrm{m}$, i.e., at $500 \mathrm{~m}$ distance in the $x$.

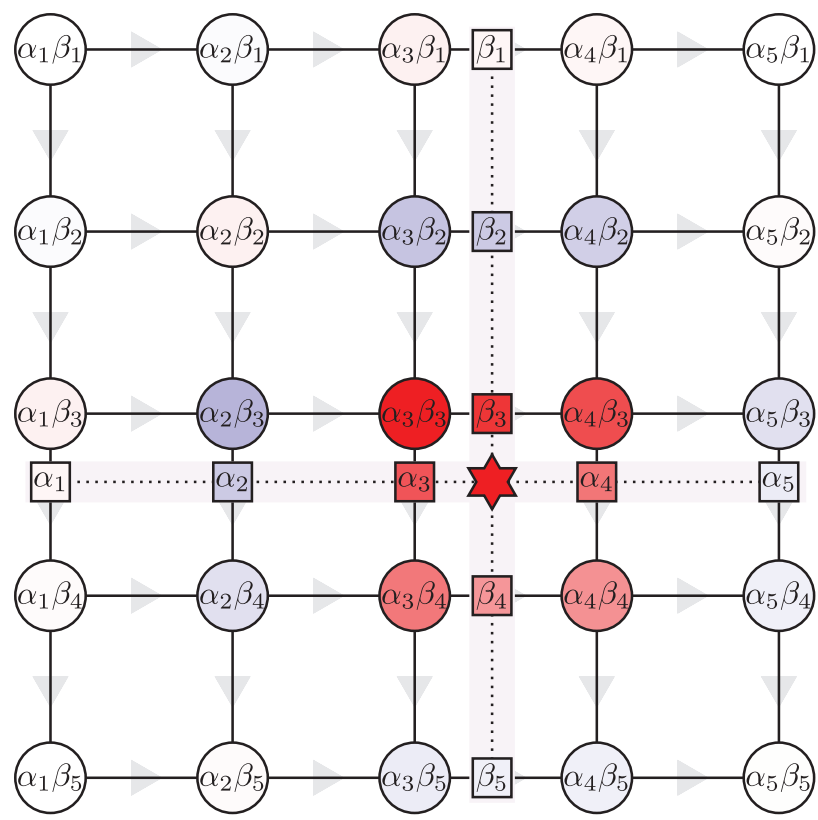

Figure 5. An arbitrarily located point source (the red star) is sampled onto a regular FD simulation by injecting its contribution into a grid of $5 \times 5$ surrounding pressure nodes (circles, colored to reflect a positive [red] or negative [blue] value). The cross-combination of two 1D interpolating FD stencils $\left(\alpha_{l}\right.$ and $\beta_{l}$, drawn with colored squares) gives the weights for the injection. Essentially, the original pointsource is distributed over a "source-array" of 25 nodes.

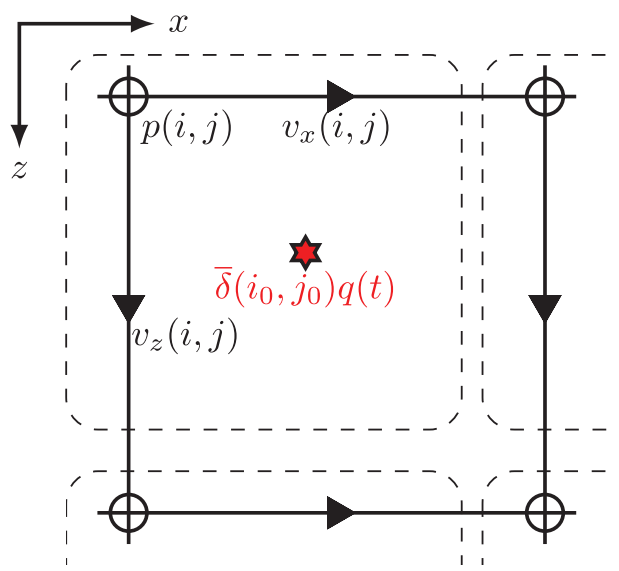

Figure 6. The discretized FD grid for wave simulations. The locations correspond to a reference grid where $p(i, j)=p(i \Delta x, j \Delta z)$ and staggered grid where $v_{x}(i, j)=v_{x}((i+1 / 2) \Delta x, j \Delta z)$ and $v_{z}(i, j)=v_{z}(i \Delta x,(j+1 / 2) \Delta z)$. A source, denoted by the red star, is arbitrarily placed within the FD grid. 
and $z$-directions. In a second simulation, the source and receiver are translated with increment $\Delta z / 2$ and $\Delta x / 2$. In the second simulation, we inject the source on a $9 \times 9$ grid with the top-left $(x, z)=$ $(1160,150) \mathrm{m}$ to bottom-right $(x, z)=(1240,230) \mathrm{m}$. We record the wavefield on a $15 \times 15$ grid with the top-left $(x, z)=(1630,620) \mathrm{m}$ and bottom-right $(x, z)=(1770,760) \mathrm{m}$. We compare results using Taylor coefficients to those of five other operators optimized up to $K_{c}=\pi / 2$. For Hicks' (2002) weights, that means using a Kaiser window with parameter $b=6.31$ on the source and $b=10.95$ on the receiver (the values are taken from Table 1 in Hicks, 2002). The coefficients and resulting simulations may be reproduced with the provided MATLAB script example_seismic_injection.m.

The FD operators for the source injections are plotted against the cost functions in Figure 7. The main point to note is that the operator due to Hicks (2002) has a relatively large error compared to the other operators. However, the optimal group-velocity weights also show a nonnegligible error, particularly for the low wavenumbers. Conversely, the weights designed for the relative error maintain a small error over the entire wavenumber range.

Figure 8 shows the recorded time series. The simulations took an average of $21 \mathrm{~s}$ to run, whereas the FD operators were created within at most $0.03 \mathrm{~s}$. In a close-up, we observe that significant differences in amplitude are present. In addition, we show the root-mean-square (rms) error from $t=0 \mathrm{~s}$ to $t=0.54 \mathrm{~s}$, as well as the maximum error over this range. The Taylor coefficients lead to the worst performance of all methods. The method from Hicks (2002) yields much better performance, and the optimized FD operators for the relative cost function result in an additional reduction of the rms and maximum error by a factor of approximately 2 . Interestingly, the operator optimized with the group-velocity cost function does not reflect such
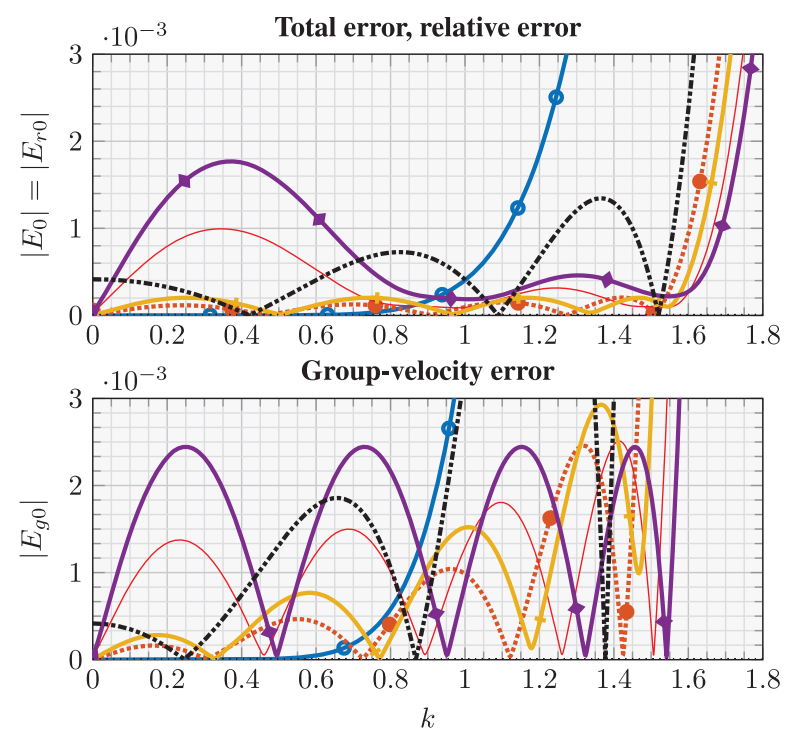

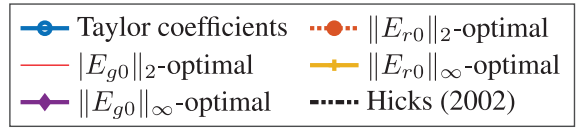

Figure 7. Error functions for six designed FD operators to approximate interpolation, sampled at $\Delta_{l}=\{-4.5,-3.5, \ldots, 3.5\}$ to inject a point source in a FD simulation. Except the Taylor coefficients, all operators were designed to minimize the error up to a critical wavenumber $K_{c}=\pi / 2$. For interpolation $(n=0)$, the total error $E_{0}$ and relative error $E_{r 0}$ are identical. gains, and the equiripple operator actually performs worse than the operator due to Hicks (2002). This corroborates Hicks' (2002) assertion that an optimal solution for the total cost function is sufficient, and that the group-velocity error should not be optimized.

\section{DISCUSSION}

The FD operators designed for the relative error and the groupvelocity error produced different results. Notably, although the operator designed for the relative error produced optimal results in all three examples, the operator designed for the group-velocity criterion did less so. This latter observation was surprising because the group-velocity optimal operators lead to a flat relative error response and are widely used as optimal modeling weights (e.g., as options in the utility package Seismic Unix [Stockwell and Cohen, 2008] or as preset coefficients with the open-source viscoelastic modeling engine SOFI2D [Bohlen et al., 2016]). The precise reason for the reduced performance in the modeling and injection examples is difficult to pin down. Potentially, it is just the fact that the group-velocity cost function is more "strict" compared to the relative cost function. For a given bandwidth, the weights for the group-velocity cost function thus result in a larger relative error than the weights for the relative cost function, or, conversely, they cover a smaller bandwidth for a given maximum error. Therefore, the coefficients for the relative cost
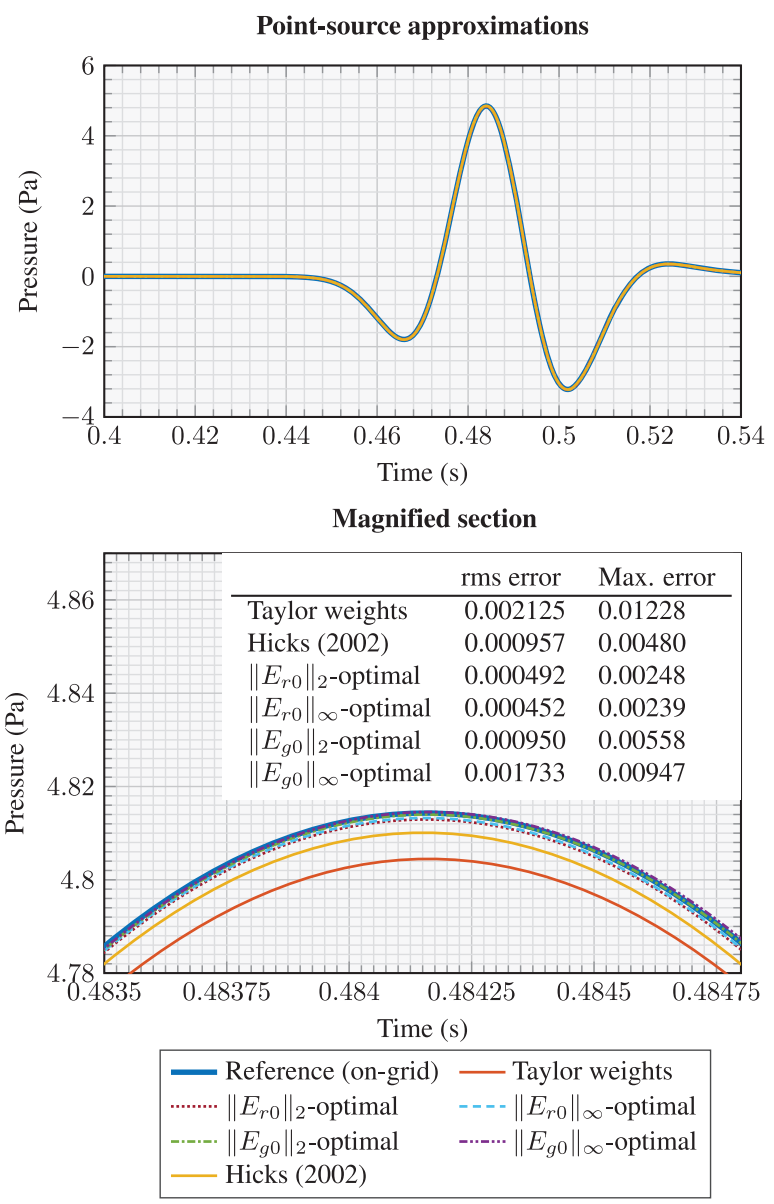

Figure 8. Comparison between a simulation where the source and receiver are located on the grid to six simulations where the sources and receivers are offset from the grid. 
function reach the best solution: covering the largest relative error bandwidth with a sufficiently small error over the entire bandwidth.

Finally, we are not aware of any other work in the literature in which a comparison of the performance of the various cost functions and optimality criteria has been made. Research papers about FD coefficients tend to demonstrate their results in (band-limited) wave simulations. In such cases, the Taylor coefficients, which are optimal for polynomials but less so for band-limited data, are easily outperformed. With the supplemented MATLAB scripts, it will be possible for other authors to study which cost function and which optimality condition yield the optimal FD operators for various problems - not just limited to the test cases provided here. Future work can similarly investigate the applicability of these FD operators for irregular grids. Compared to, e.g., Opršal and Zahradník (1999), the FD operators can then be extended to arbitrarily highorder accuracy. The one-sided operators at the boundary can also be further investigated to model a simple free surface for acoustic (Berland et al., 2007) and elastic wave simulations (Tarrass et al., 2011).

\section{CONCLUSION}

We presented a general method to obtain equiripple and leastsquares optimal FD operators. These operators weigh samples at arbitrary offsets and allow for the computation of derivatives of arbitrary orders. We assessed three cost functions: (1) the total error, (2) the relative error, and (3) the group-velocity error. We found the equiripple solution through a complex extension of the Remez exchange algorithm. We found the least-squares solution in an overdetermined first iteration of the algorithm. The described methodology is valid for arbitrary orders of differentiation and arbitrary sampling locations. It can, thus, be used to design operators for a wide range of applications beyond the examples described in this paper. The results obtained for the three examples (accurate FD modeling of wave propagation on regular and irregular grids and injecting and recording waves at arbitrary locations not coinciding with the grid) suggest that the most accurate results are obtained with weights designed for the relative error. The equiripple FD operators led to approximately $4 \%-10 \%$ smaller errors compared to the least-squares FD operators.

\section{ACKNOWLEDGMENTS}

This work was supported by SNF grant no. 2-77220-15. We thank the editors J. Dellinger and J. Shragge as well as three anonymous reviewers for helpful comments that improved the manuscript and the reproducibility of the code.

\section{DATA AND MATERIALS AVAILABILITY}

Data associated with this research are available and can be accessed via the following URL: http://software.seg.org/2020/0004.

\section{APPENDIX A}

\section{FURTHER DETAILS ON THE REMEZ EXCHANGE ALGORITHM}

\section{The number of maxima}

Equation 19 has $L+m-1+m+1$ unknowns (being the $L$ FD weights, the $m$ maximum wavenumbers except the knowledge that $k_{m}=K_{c}$, the $m$ error phases, and the constant error magnitude) and only $2 m$ equations. However, we know that $m-1$ maxima are extrema (i.e., peaks) of the total error function - which provides a further $2 m-1$ constraints. Only the final wavenumber $k_{m}=K_{c}$ is not an extremum and thus does not provide a further constraint. Combining the unknowns and constraints, $L+2 m \leq 4 m-1$ requires $m \geq\lceil(L+1) / 2\rceil$ maxima to obtain a well-posed problem, where $\lceil\cdot\rceil$ rounds to the nearest integer above. For the relative and group-velocity cost functions, the additional constraints of equation 14 lower this number to $m \geq\lceil(L+1-n) / 2\rceil$. In our experience, the optimal solutions were found with the minimum number of $m$ allowed, thus interpreting $\geq$ as a strict equality.

We can point out two special cases that arise when designing symmetric FD operators:

1) Even-order derivatives $(n=0,2, \ldots)$ with an odd number of sampling points (e.g., $\Delta_{l}=\{-1,0,1\}$ ) allow an arbitrary additional sampling point without altering the operator. We thus find the coefficients to be the same if we sample an additional point, illustrated with the Taylor coefficients for $n=2$ :

$$
\alpha= \begin{cases}\{1,-2,1\} & \text { for } \Delta_{l}=\{-1,0,1\}, \\ \{1,-2,1,0\} & \text { for } \Delta_{l}=\{-1,0,1,2\} .\end{cases}
$$

2) Odd-order derivatives $(n=1,3, \ldots)$, with an even number of sampling points (e.g., $\Delta_{l}=\{-0.5,0.5\}$ ) allow an arbitrary additional sampling point without altering the operator. We thus find the coefficients to be the same if we sample an additional point, illustrated with the Taylor coefficients for $n=1$ :

$$
\alpha= \begin{cases}\{-1,1\} & \text { for } \Delta_{l}=\{-0.5,0.5\} \\ \{-1,1,0\} & \text { for } \Delta_{l}=\{-0.5,0.5,1.5\}\end{cases}
$$

These two exceptions to the rule yield $L \rightarrow L+1$, as we could add one additional sampling point without influencing the operator. These exceptions influence the value of $m$ for odd-order derivatives with an even number of symmetric sampling points or an even-order derivative with an odd number of symmetric sampling points. This is visible, for example, for the symmetric FD operators in Figure 1 that use 14 points to approximate the first-order derivative. In the figure, we see that eight maxima are achieved. The formula for the number of maxima suggests one less: $\lceil(14+1-1) / 2\rceil=7$. However, we obtain eight maxima by seeing that we can increase $L$ by one to mimic one arbitrary additional point: $\lceil(14+1+1-1) / 2\rceil=8$.

\section{Graphical example of iterations}

We provide a small graphical explanation of one run of the Remez exchange algorithm in Figure A-1; the converged solution can be found using the MATLAB files with command FD_gV $([-5.2: 2.8], 1,1,0,-1)$. The -1 argument at the end leads to a solution that is not initialized with the expected maxima from an $L^{2}$ inversion first. At every iteration, the maxima of the absolute error-function $\left|E_{g 1}\right|$ can be found by interpolating the function for all $k \in\left[0, K_{c}\right]$. The $m$ (local) maxima of $\left|E_{g 1}\right|$ can correspondingly be obtained with any algorithm. We used a MATLAB one-liner to find the maxima of $E$ in the $n$ interpolated values, i.e., where the value is higher on the left- and right-hand side except for the edges: find $(E>[E(1)-1 ; E(1: n-1)] \& E>[E(2: n) ; E(n)-1])$. We can easily enforce, furthermore, that the final wavenumber is a maxi- 

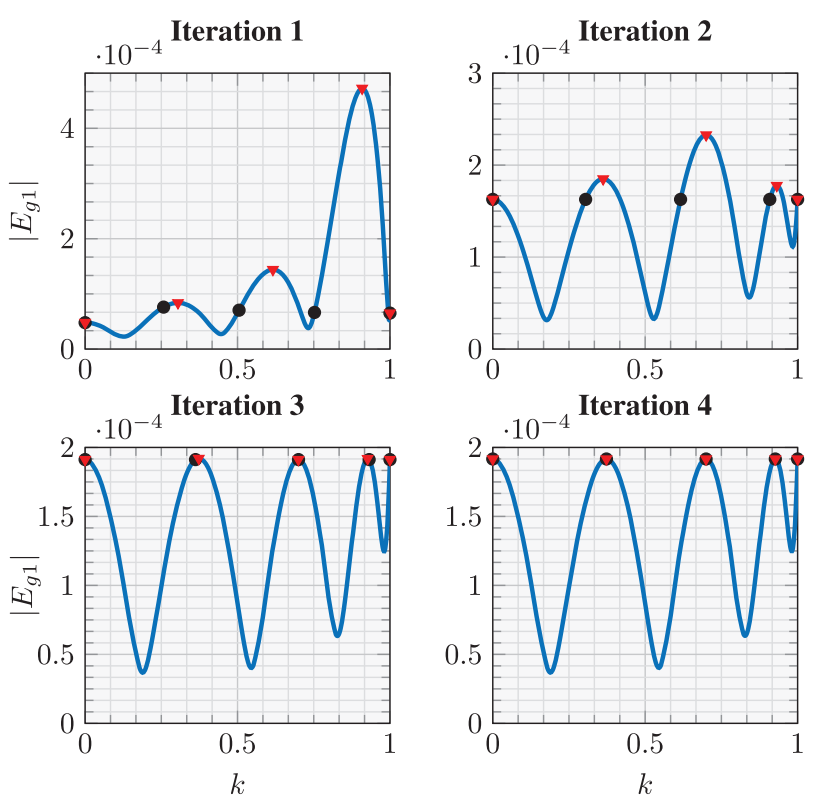

Figure A-1. Graphical example of the Remez exchange algorithm. The sample locations are $\Delta_{l}=\{-5.2,-4.2, \ldots, 2.8\}$, the error function is $E_{g 1}(k)$, i.e., the group-velocity error of the first-order derivative, the critical wavenumber $K_{c}=1$. At every iteration, FD coefficients are generated with the assumption that the maxima lie at the black circles. The actual maxima are then determined from a plot of $\left|E_{g 1}(k)\right|$, using the obtained FD coefficients, here shown with red triangles. The red triangles are exchanged to become the new set of maxima for the next iteration. The algorithm converges after five iterations, in $0.046 \mathrm{~s}$.

mum, $k_{m}=K_{c}$. The angles $\theta$ are then extracted at all local maxima and are used for the next iteration.

\section{APPENDIX B \\ RELATIVE AND GROUP-VELOCITY COST FUNCTIONS}

We can provide a quantitative as well as a qualitative description of the relative and group-velocity cost functions. We first relate the cost functions to the one- and two-way wave equations for which the relative and group-velocity cost functions naturally emerge when we use an FD approximation for the spatial derivatives. Furthermore, we show that the maximum group-velocity error of an FD operator is always larger than the maximum relative error.

\section{One-way wave equation}

Assume a one-way wave equation in one dimension with velocity $c_{0}$, where subscripts $x$ and $t$ differentiate $u$ to $x$ and $t$, respectively,

$$
c_{0}=\frac{u_{t}}{u_{x}}
$$

with a plane-wave ansatz $u=e^{i \omega t+i k x}$ for $k=\omega / c_{\mathrm{ph}}$. This defines the phase velocity

$$
c_{\mathrm{ph}}=\frac{\omega}{k}
$$

and the group velocity

$$
c_{\mathrm{gr}}=\frac{\mathrm{d} \omega}{\mathrm{d} k}=c_{\mathrm{ph}}+k \frac{\mathrm{d} c_{\mathrm{ph}}}{\mathrm{d} k} .
$$

The ansatz for equation B-1 gives the analytical relation

$$
c_{0}=\frac{u_{t}}{u_{x}}=\frac{i \omega u}{i k u}=c_{\mathrm{ph}},
$$

such that all definitions are equal: $c_{\mathrm{ph}}=c_{\mathrm{gr}}=c_{0}$. However, when we use an FD approximation to compute $u_{x}$, we find

$$
c_{0}=\frac{i \omega u}{\sum_{l=1}^{L} \alpha_{l} e^{i k \Delta x_{l} u}}=\frac{i \omega}{i k\left(1-E_{r 1}(k)\right)}=\frac{c_{\mathrm{ph}}}{1-E_{r 1}(k)},
$$

where we used the relative error function from equation 12 . The phase velocity in the FD model can, thus, be expressed as

$$
c_{\mathrm{ph}}=c_{0}\left(1-E_{r 1}(k)\right) \text {, }
$$

and the group velocity correspondingly as

$$
\begin{aligned}
c_{\mathrm{gr}}(k) & =c_{\mathrm{ph}}+k \frac{\mathrm{d} c_{\mathrm{ph}}}{\mathrm{d} k}, \\
& =c_{0}\left(1-E_{r 1}(k)-k \frac{\mathrm{d} E_{r 1}(k)}{\mathrm{d} k}\right), \\
& =c_{0}\left(1-E_{g 1}(k)\right),
\end{aligned}
$$

where we used the group-velocity error from equation 15 .

We can, thus, view the relative error $E_{r 1}(k)$ and the group-velocity error $E_{g 1}(k)$ as a systematic alteration of the desired phase and group velocities of the physical model. We can thus qualitatively interpret a maximum relative error of, e.g., $E_{r 1}=10^{-4}$ as a $0.01 \%$ maximum error in the modeled phase velocities. The same goes for the group-velocity error.

\section{Two-way wave equation}

Assume a two-way wave equation with velocity $c_{0}$,

$$
c_{0}^{2}=\frac{u_{t t}}{u_{x x}},
$$

with the same plane-wave ansatz as in the previous section. We then obtain

$$
c_{0}^{2}=\frac{u_{t t}}{u_{x x}}=\frac{-\omega^{2}}{-k^{2}}=c_{\mathrm{ph}}^{2}
$$

and again realize that $c_{\mathrm{ph}}=c_{\mathrm{gr}}=c_{0}$ in the analytic case. If we use FDs to compute the spatial derivative $u_{x x}$, we find

$$
c_{0}^{2}=\frac{-\omega^{2} u}{\sum_{l=1}^{L} \alpha_{l} e^{i k \Delta x_{l}} u}=\frac{-\omega^{2}}{-k^{2}\left(1-E_{r 2}(k)\right)}=\frac{c_{\mathrm{ph}}^{2}}{1-E_{r 2}(k)} .
$$


The phase velocity in the FD model is thus

$$
c_{\mathrm{ph}}^{2}=c_{0}^{2}\left(1-E_{r 2}(k)\right)
$$

and the group velocity correspondingly is

$$
\begin{aligned}
c_{\mathrm{gr}}^{2}(k) & =c_{0}^{2}\left(1-E_{g 2}(k)+\frac{k^{2}}{4-4 E_{r 2}(k)}\left(\frac{\mathrm{d} E_{r 2}}{\mathrm{~d} k}\right)^{2}\right), \\
& \approx c_{0}^{2}\left(1-E_{g 2}(k)\right),
\end{aligned}
$$

where we neglect the squared-order error term.

As in the previous section, we can observe that the phase and group velocities are modeled at the wrong speeds due to using an FD approximation for the spatial derivatives, and we can express these errors in terms of the corresponding cost functions. Hence, for the first- and second-order FD models of seismic waves, it is essential to minimize the relative or group-velocity cost function if we desire small phase- or group-velocity errors.

\section{Maximum error bounds}

For any FD operator and wavenumber range from zero up to an arbitrary wavenumber $K$, we pose that

$$
k \in[0, K], \quad\left\|E_{r n}(k)\right\|_{\infty} \leq\left\|E_{g n}(k)\right\|_{\infty},
$$

meaning that the maximum error in the group-velocity cost function is always larger than or equal to the maximum error in the relative cost function. We can show this property by expressing the groupvelocity cost function in terms of the relative cost function,

$$
\left\|E_{g n}(k)\right\|_{\infty}=\left\|E_{r n}(k)+k \frac{\mathrm{d} E_{r n}(k)}{\mathrm{d} k}\right\|_{\infty} .
$$

Because $E_{r n}(k)$ is an oscillating function, we are furthermore guaranteed that $E_{r n}(k)$ and $\mathrm{d} E_{r n} / \mathrm{d} k$ have the same sign leading up to any extremum of $\left|E_{r n}\right|$. Leading up to any extremum, we thus have

$$
\left\|E_{g n}(k)\right\|_{\infty}=\left\|E_{r n}(k)\right\|_{\infty}+\left\|k \frac{\mathrm{d} E_{r n}(k)}{\mathrm{d} k}\right\|_{\infty} .
$$

Equation B-17 implies that equation B-15 must hold because while $\left|E_{r n}\right|$ approaches any maximum, $\left|E_{r n}\right|+\left|k \mathrm{~d} E_{r n} / \mathrm{d} k\right|$ is growing at least as fast.

The consequence of equation B-15 is that an optimal solution for the group-velocity cost function also automatically bounds its maximum relative error.

\section{REFERENCES}

Alkhairy, A., 1994, A complex Chebyshev approximation algorithm for FIR filter design: International Conference on Acoustics, Speech, and Signal Processing, ICASSP-94, IEEE, III-549.

Berland, J., C. Bogey, O. Marsden, and C. Bailly, 2007, High-order, low dispersive and low dissipative explicit schemes for multiple-scale and boundary problems: Journal of Computational Physics, 224, 637-662, doi: $10.1016 /$ j.jcp.2006.10.017.

Bohlen, T., D. De Nil, D. Köhn, and S. Jetschny, 2016, SOFI2D seismic modeling with finite differences: $2 \mathrm{D}$ - elastic and viscoelastic version: Karlsruhe Institute of Technology.

Bowen, M., and R. Smith, 2005, Derivative formulae and errors for non-uniformly spaced points: Proceedings of the Royal Society of London A: Mathematical, Physical and Engineering Sciences, 461, 1975-1997, doi: $10.1098 /$ rspa.2004.1430.
Chu, C., and P. L. Stoffa, 2012, Determination of finite-difference weights using scaled binomial windows: Geophysics, 77, no. 3, W17-W26, doi: 10.1190/geo2011-0336.1.

Crase, E., 1990, High-order (space and time) finite-difference modeling of the elastic wave equation: 60th Annual International Meeting, SEG, Expanded Abstracts, 987-991, doi: 10.1190/1.1890407.

Dablain, M., 1986, The application of high-order differencing to the scalar wave equation: Geophysics, 51, 54-66, doi: 10.1190/1.1442040.

Fornberg, B., 1988, Generation of finite difference formulas on arbitrarily spaced grids: Mathematics of Computation, 51, 699-706, doi: 10.1090/ S0025-5718-1988-0935077-0.

Fornberg, B., 1990, High-order finite differences and the pseudospectral method on staggered grids: SIAM Journal on Numerical Analysis, 27, 904-918, doi: 10.1137/0727052.

Fornberg, B., 1998, Classroom note: Calculation of weights in finite difference formulas: SIAM Review, 40, 685-691, doi: 10.1137/ S0036144596322507.

Hayashi, K., and D. R. Burns, 1999, Variable grid finite-difference modeling including surface topography: 69th Annual International Meeting, SEG, Expanded Abstracts, 528-531, doi: 10.1190/1.1821071.

He, Z., J. Zhang, and Z. Yao, 2019, Determining the optimal coefficients of the explicit finite-difference scheme using the Remez exchange algorithm: Geophysics, 84, no. 3, S137-S147, doi: 10.1190/geo2018-0446.1.

Hicks, G. J., 2002, Arbitrary source and receiver positioning in finite-difference schemes using Kaiser windowed sinc functions: Geophysics, 67, 156-165, doi: 10.1190/1.1451454.

Holberg, O., 1987, Computational aspects of the choice of operator and sampling interval for numerical differentiation in large-scale simulation of wave phenomena: Geophysical Prospecting, 35, 629-655, doi: 10 .1111/j.1365-2478.1987.tb00841.x.

Ikelle, L. T., and L. Amundsen, 2018, Introduction to petroleum seismology: SEG.

Khan, I. R., and R. Ohba, 2000, New finite difference formulas for numerical differentiation: Journal of Computational and Applied Mathematics, 126 269-276, doi: 10.1016/S0377-0427(99)00358-1.

Kindelan, M., A. Kamel, and P. Sguazzero, 1990, On the construction and efficiency of staggered numerical differentiators for the wave equation: Geophysics, 55, 107-110, doi: 10.1190/1.1442763.

Kindelan, M., M. Moscoso, and P. Gonzalez-Rodriguez, 2016, Optimized finite difference formulas for accurate high frequency components: Mathematical Problems in Engineering, 2016, 1-15, doi: 10.1155/2016/ 7860618.

Koene, E. F. M., J. O. A. Robertsson, F. Broggini, and F. Andersson, 2018, Eliminating time dispersion from seismic wave modeling: Geophysical Journal International, 213, 169-180, doi: 10.1093/gji/ggx563.

Kosloff, D., R. C. Pestana, and H. Tal-Ezer, 2010, Acoustic and elastic numerical wave simulations by recursive spatial derivative operators: Geophysics, 75, no. 6, T167-T174, doi: 10.1190/1.3485217.

Lele, S. K., 1992, Compact finite difference schemes with spectral-like resolution: Journal of Computational Physics, 103, 16-42, doi: 10.1016/ 0021-9991(92)90324-R.

Levander, A. R., 1988, Fourth-order finite-difference P-SV seismograms: Geophysics, 53, 1425-1436, doi: 10.1190/1.1442422.

Liu, Y., 2013, Globally optimal finite-difference schemes based on least squares: Geophysics, 78, no. 4, T113-T132, doi: 10.1190/geo2012-0480.1.

Liu, Y., 2014, Optimal staggered-grid finite-difference schemes based on least-squares for wave equation modelling: Geophysical Journal International, 197, 1033-1047, doi: 10.1093/gji/ggu032.

Liu, Y., and M. K. Sen, 2009, A practical implicit finite-difference method: Examples from seismic modelling: Journal of Geophysics and Engineering, 6, 231-249, doi: 10.1088/1742-2132/6/3/003.

Marvasti, F., 2001, Nonuniform sampling: Theory and practice: Springer US.

MATLAB, 2017, Version 9.2.0 (r2017a): The MathWorks Inc.

McClellan, J., T. Parks, and L. Rabiner, 1973, A computer program for designing optimum FIR linear phase digital filters: IEEE Transactions on Audio and Electroacoustics, 21, 506-526, doi: 10.1109/TAU.1973.1162525.

Mittet, R., 2017, On the internal interfaces in finite-difference schemes: Geophysics, 82, no. 4, T159-T182, doi: 10.1190/geo2016-0477.1.

Mittet, R., and B. Arntsen, 1999, General source and receiver positions in coarse-grid finite-difference schemes: 69th Annual International Meeting, SEG, Expanded Abstracts, 1855-1858, doi: 10.1190/1.1820906.

Mittet, R., and B. Arntsen, 2000, General source and receiver positions in coarse-grid finite-difference schemes: Journal of Seismic Exploration, 9, 73-92.

Opršal, I., and J. Zahradník, 1999, Elastic finite-difference method for irregular grids: Geophysics, 64, 240-250, doi: 10.1190/1.1444520.

Özdenvar, T., and G. A. McMechan, 1996, Causes and reduction of numerical artefacts in pseudo-spectral wavefield extrapolation: Geophysical Journal International, 126, 819-828, doi: 10.1111/j.1365-246X.1996.tb04705.x.

Preuss, K., 1987, A novel approach for complex Chebyshev-approximation with FIR filters using the Remez exchange algorithm: International 
Conference on Acoustics, Speech, and Signal Processing ICASSP'87, IEEE, 872-875.

Remez, E. Y., 1934, Sur la détermination des polynômes d'approximation de degré donnée: Communications of the Kharkov Mathematical Society, 10, 41-63.

Robertsson, J. O., I. Moore, M. Vassallo, K. Özdemir, D.-J. van Manen, and A. Ozbek, 2008, On the use of multicomponent streamer recordings for reconstruction of pressure wavefields in the crossline direction: Geophysics, 73, no. 5, A45-A49, doi: 10.1190/1.2953338.

Schulist, M., 1990, Improvements of a complex FIR filter design algorithm: Signal Processing, 20, 81-90, doi: 10.1016/0165-1684(90)90078-D

Shragge, J., and B. Tapley, 2017, Solving the tensorial 3D acoustic wave equation: A mimetic finite-difference time-domain approach: Geophysics, 82, no. 4, T183-T196, doi: 10.1190/geo2016-0691.1.

Stockwell, J. W., Jr., and J. K. Cohen, 2008, The new SU user's manual: Center for wave phenomena, Colorado School of Mines.

Stork, C., 2013, Eliminating nearly all dispersion error from FD modeling and RTM with minimal cost increase: 75th Conference and Exhibition, EAGE, Extended Abstracts, doi: 10.3997/2214-4609.20130478.

Sun, W., B. Zhou, and L.-Y. Fu, 2015, A staggered-grid convolutional differentiator for elastic wave modelling: Journal of Computational Physics, 301, 59-76, doi: 10.1016/j.jcp.2015.08.017.
Tarrass, I., L. Giraud, and P. Thore, 2011, New curvilinear scheme for elastic wave propagation in presence of curved topography: Geophysical Prospecting, 59, 889-906, doi: 10.1111/j.1365-2478.2011.00972.x.

Virieux, J., 1986, P-SV wave propagation in heterogeneous media: Velocitystress finite-difference method: Geophysics, 51, 889-901, doi: 10.1190/1 .1442147 .

Yang, L., H. Yan, and H. Liu, 2017, Optimal staggered-grid finite-difference schemes based on the minimax approximation method with the Remez algorithm: Geophysics, 82, no. 1, T27-T42, doi: 10.1190/geo2016-0171.1.

Zhang, J.-H., and Z.-X. Yao, 2013a, Optimized explicit finite-difference schemes for spatial derivatives using maximum norm: Journal of Computational Physics, 250, 511-526, doi: 10.1016/j.jcp.2013.04.029.

Zhang, J.-H., and Z.-X. Yao, 2013b, Optimized finite-difference operator for broadband seismic wave modeling: Geophysics, 78, no. 1, A13-A18, doi: 10.1190/geo2012-0277.1.

Zhi-Yang, W., L. Hong, T. Xiang-De, and W. Yang, 2015, Optimized finitedifference operator based on Chebyshev auto-convolution combined window function: Chinese Journal of Geophysics, 58, 192-206, doi: 10.1002/ cjg2.20166.

Zhou, B., and S. Greenhalgh, 1992, Seismic scalar wave equation modeling by a convolutional differentiator: Bulletin of the Seismological Society of America, 82, 289-303. 